\title{
29. THE MOTION OF HALLEY'S COMET FROM 837 TO 1910
}

\author{
J. L. BRADY
}

Lawrence Radiation Laboratory, University of California, Livermore, Calif., U.S.A.

\begin{abstract}
Numerical experiments have been made in an attempt to remove the residuals of $\mathrm{P} /$ Halley and link the seven apparitions from 1456 to 1910 . All efforts to link more than two apparitions using Newtonian equations have invariably failed. However, by the addition of a secular term to the equations of motion, the four apparitions from 1910 back to 1682 can be linked by a numerical integration which represents the observations to contemporary accuracy. When this integration is continued, the apparitions of 1607,1531 , and 1456 show residuals of less than one day in the time of perihelion passage. Prior to 1456 the residuals begin to run off but, with the exception of 1222 and 1066, the apparitions back to 837 show residuals no greater than four days in the time of perihelion passage. The residuals of 20 days in 1222 and 7 days in 1066 appear anomalous but can be made reasonable if the Chinese records are adopted in preference to the European records.
\end{abstract}

